\title{
Potentiality of magnetoelectric composites for Wireless Power Transmission in medical implants
}

\author{
Giulia Rizzo ${ }^{1,2}$, Vincent Loyau ${ }^{3}$, Ronald Nocua ${ }^{1}$, Jean Christophe Lourme ${ }^{1}$, Elie Lefeuvre ${ }^{2}$ \\ 1 -ValoTec, 1 Mail du Prosfesseur Georges Mathé, 94800 Villejuif, France \\ 2 - C2N, Université Paris-Sud, Avenue de la Vauve, 91200 Palaiseau, France \\ 3 - SATIE, Ecole Normale Supérieure Paris-Saclay, 94230 Cachan, France \\ Email : giulia.rizzo@valotec.com
}

\begin{abstract}
Electric power can be wirelessly transferred to power supply medical devices, in order to reduce the size of the implants and increase their lifetime. The inductive power transmission, through the interaction between two coils, is compared to a novel magnetoelectric (ME) technology. In this case, the external coil delivers the energy to a ME receiver, made with an electroactive (e.g piezoelectric) material, bonded between two magnetostrictive layers.

This new technology can overpass the issues of directionality and heating, present in the inductive transmission technique, and open new prospective for battery-less medical implants.
\end{abstract}

Keywords - Magnetostrictive material, piezoelectric, wireless power transfer, in-vivo power, magnetoelectric effect

\section{INTRODUCTION}

Nowadays active implantable medical devices (AIMD) play an important role in monitoring, diagnosing and treating patients. For this reason, device manufacturers invent smart and autonomous implants that improve the patients' health and life quality [1].

Since the initial demonstration, in the late 1950s, most of the implantable electronic devices, used in clinical circumstances, have been powered by batteries. However, the use of batteries imposes several limitations to the current AIMD. First, due to the finite lifetime of batteries, surgeries are often required, for replacing the discharged units. Second, while electronic circuits get smaller dimensions with rapid development of semiconductor integrated circuit (IC) technologies, the volumetric capacity of batteries has improved rather slowly in the past few decades. Third, the battery has an impact also in the weight of the implantable system, which becomes a major hurdle in minimizing the overall device's size. In addition, even though AIMD are tightly sealed with biocompatible encapsulation materials, there is a finite risk for leakage of electrolytes from the battery, which may cause toxic effects and inflammatory reaction in tissues [2].

Following these issues, researchers have been studying wireless power transmission (WPT) techniques for decades. In this case, an external source is used to transmit energy, for example acoustic [3] or electromagnetic [4], from out-body to in-body. The internal block converts the received energy into power to supply the device. Both two approaches have positive and negative aspects. In general, the advantages of the WPT is the constant availability of the energy source, instantaneous transmission and high rate of delivered power. The disadvantages are limited at the device's comfortability for the patient and the Specific Absorption Rate (SAR) constraint, imposed by the medical regulatory [5]. The main parts of the AIMD are made by a metallic packaging, to ensure the system's biocompatibility and the device's longterm hermeticity. Using WPT can cause the heating of the enclosure and so a consequent heating of the patient's body.

The objective of this paper is the investigation of an alternative way to wireless charge a medical implant. In general, the most employed WPT technique is based on an inductive electromagnetic system [4], which delivers the energy from a transmitter out-body coil to another one, inserted in the medical implant. On the contrary, the proposed solution consists in the replacement of the internal coil with a hybrid system, formed by two layers of magnetostrictive materials and a piezoelectric (PZT) layer in the middle.

The piezoelectric (PZT) materials can become electrically polarised, in response to an applied mechanical stress. As the inverse effect, they show a mechanical strain, when placed in an external electric field [6]. PZTs work along several modes, each one is described through its own coupling coefficient $\mathrm{k}$. This coefficient qualifies the efficiency of energy conversion between electrical and mechanical. If strain and polarization are in the same direction, the $\mathrm{k}_{33}$ mode is defined. When strain and polarization are orthogonal, the $\mathrm{k}_{31}$ mode is considered, even if it results less effective than $\mathrm{k}_{33}$ [6].

On the contrary, the magnetostrictive materials can deform their structure, in response to a change in their internal magnetization. This variation can be brought either by a change in temperature or by the application of a magnetic field [7]. Thanks to Villari effect, it is possible to have the inverse relationship, so obtaining a change of sample's magnetization when a mechanical stress is applied to the system [8]. The effects can be linear or non-linear with respect to the external fields [9].

The parameter, which describes better the behaviour of the entire hybrid system, is defined as magnetoelectric effect. It refers to the electric polarization induced by an applied magnetic field, or the magnetization induced by an applied electric field [9]. In ambient temperature, since single-phase ME materials are not suitable for industrial applications, due to their generally low ME coefficient, the development of multi-phase ME composite materials is tested.

\section{MATERIALS}

In this paper, two systems are considered and compared. The first one (Fig 1.A) is based on a near field 
transmission technique, which can be used for energy delivery [7] and for communications [10]. It consists on the wireless interaction between two coils connected to resonant circuits, the receiver $(\mathrm{Rx})$ and the transmitter (Tx) units. $\mathrm{Rx}$ is usually inserted inside the medical device, permanently inbody, and the source Tx is outside the body, in order to be switchable, up to patients' needs.

The second example (Fig 1.B) is the innovative technology, based on a magnetoelectric composite. The new system converts the electromagnetic energy, generated from the external transmitter coil, in mechanical strain thanks to the magnetostrictive layers. From the elastic stress, the piezoelectric material converts this mechanical energy in electrical energy. The combination of magnetostrictive and piezoelectric materials allows to convert the magnetic energy into electric, through the modification of elastic bonding, exhibiting new properties that do not belong of its individual constituent phases [9]. The innovation of this technology can overpass the directionality and distance constraints of the first case.

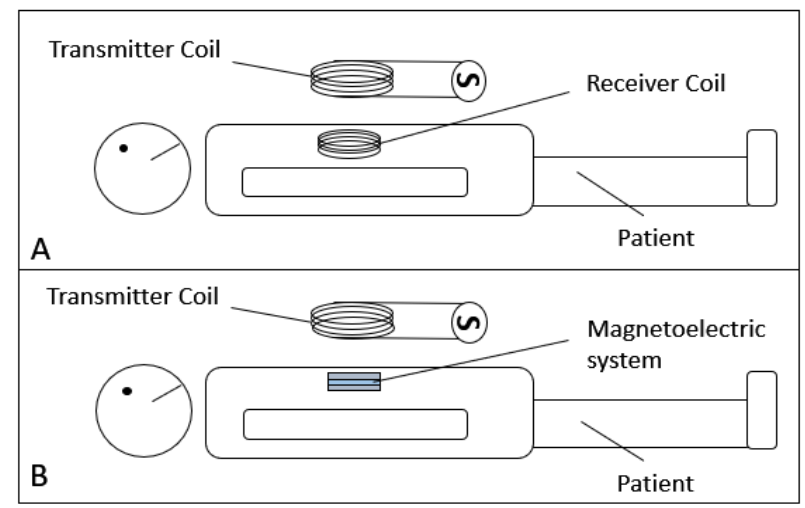

Fig.1A WPT through induction between two coil.

Fig. 1B through the magnetoelectric composite.

The most employed magnetostrictive materials are Terfenol-D [14] and MetGlas [15], with respectively saturation strains $\lambda_{\mathrm{s}}$ of $2000 \mathrm{ppm}$ (at $240 \mathrm{kA} / \mathrm{m}$ ) and $30 \mathrm{ppm}$ (at $24 \mathrm{kA} / \mathrm{m})$. This parameter is the value of the maximum achievable strain, at the corresponding saturation magnetic field. The material can be used in a laminate shape [14] or disk form [16], with a general thickness of $1 \mathrm{~mm}, 10-15 \mathrm{~mm}$ of length/diameter and $10 \mathrm{~mm}$ of width. The dimensions influence the resonance frequency of the system, which is of the order of $70-100 \mathrm{kHz}$ for the previous parameters.

For the piezoelectric layer, the commonly used materials are the piezoceramic, PZT-5 and PZT-8, with a respectively $\mathrm{k}_{33}$ of 0.74 and 0.72 and a $\mathrm{k}_{31}$ of 0.38 and 0.35 [15] or piezo-fiber composite (PFC), assembled with epoxy [17]. The difference between them is shown in the value of resonance frequency; for piezoceramic composites can be $70-100 \mathrm{kHz}$ [15] and for PFC is a bit lower, or order of 30$50 \mathrm{kHz}[17]$. In general, they should assume the same shape of the adjacent magnetostrictive materials, in order to maximize the conversion between mechanical and electrical energy.
Furthermore, the entire magnetoelectric composites can act with different polarization modes of the materials, as shown in Fig.2.

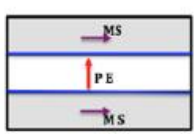

Mode LT

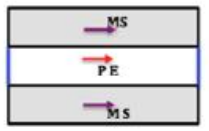

Mode LL

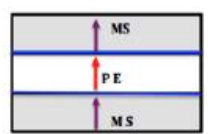

Mode TT

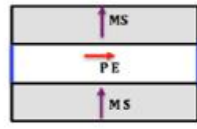

Mode TL

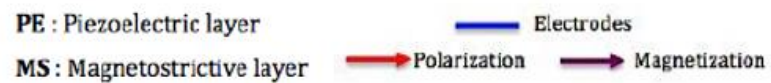

Fig. 2 Scheme different modes of polarization and magnetization for the magnetoelectric composite [12].

In L-T mode, the magnetostrictive material is magnetized along the longitudinal direction whereas the piezoelectric material is polarized along the transversal direction.

In TT-mode, the magnetostrictive material and piezoelectric material are magnetized and polarized along the transversal direction; in LL-mode along the longitudinal direction, as shown in Fig.2. The change of the mode configurations results in the mechanical stiffness values in different directions, and a consequent influence on the resonance frequency. In general, the LT configuration results the most efficient for the highest ME coefficient, but it could change for the specific applications [12].

\section{EQUIVALENT CIRCUIT}

The two systems are presented through their equivalent circuits. In both case it is necessary to work at the resonance frequency in order to increase the efficiency of the transmission. In the first case the circuit is represented in Fig. 3.

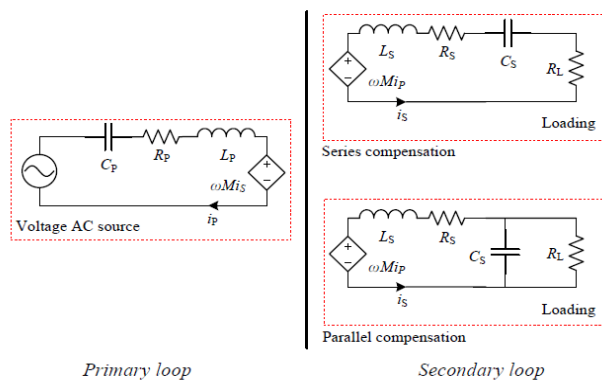

Fig.3 Equivalent circuit of inductive WPT. 
It consists in two parts, the primary block ( $\mathrm{Tx})$ and the secondary one $(\mathrm{Rx})$. Each part is formed by one coil and a resonant circuit. The transmit part consists of the power supply, Tx coil and the resonant circuit. The receiver block is constituted by the Rx coil, the resonant circuit and the load. It can have two configurations, using secondary seriesand parallel-compensations to achieve an optimal operating efficiency and a load-independent voltage-transfer ratio.

The second system is represented in Fig. 4. The equivalent generator o ${ }_{\text {in }}$ or $\mathrm{V}_{\text {in }}=\gamma \mathrm{H}_{\mathrm{ac}}$ represents the stress force due to the magnetostrictive layers as a result of the externally applied magnetic field $\mathrm{H}_{\mathrm{ac}}$. The coefficient $\gamma$ is a magnetoelectric parameter in $[\mathrm{Vm} / \mathrm{A}]$, that converts the externally applied magnetic field $\mathrm{H}_{\mathrm{ac}}$ into voltage source. The impedance $Z^{\prime}{ }_{m}=R_{m}+j \omega L_{m}+\left(j \omega C_{m}\right)^{-1}$ represents the mechanical part in which $C_{m}, L_{m}$ and $R_{m}$ are respectively the mechanical stiffness, the mass and the mechanical damping [12].

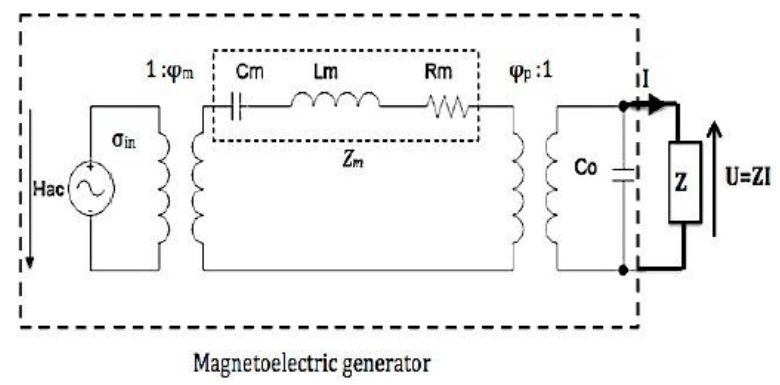

Fig.4 Equivalent circuit of WPT through magnetoelectric composite system [12].

The electrical part is represented by the clamped capacitor $\mathrm{C}_{0}$ of the piezoelectric element. The coefficients $\varphi_{\mathrm{m}}$ and $\varphi_{\mathrm{p}}$ are transformer ratios symbolizing the magnetomechanical and electromechanical damping.

\section{TRANSMITTED POWER}

From the point of view of the transmitted power, the inductive system can transfer hundreds of $\mathrm{mW}$, at resonance frequency of around $100 \mathrm{kHz}$. The amount of deliverable power is very dependent on the directionality of the two coils, their distance and the media around them. The maximum of the efficiency is shown when the two coils are coaxial and at close distance (few $\mathrm{cm}$ of distance) [11]; so it could be problematic employing this technology to charge/communicate with movable implants, i.e. leadless pacemaker [10].

In the second case, the 3-layers system at the resonance frequency of $70 \mathrm{kHz}$, can deliver an output power of $600 \mu \mathrm{W}$ with a power density of $1.4 \mu \mathrm{W} / \mathrm{mm}^{3}$ [15], employing MetGlas, Terfenol-D and PZT-5H. In another case, an RMS magnetic field-source of $1600 \mathrm{~A} / \mathrm{m}$ at $3 \mathrm{~cm}$ of distance, generates in the ME receiver, made by Terfenol-D and PFC, a power of $200 \mathrm{~mW}\left(2 \mathrm{~W} / \mathrm{cm}^{3}\right)$ [17].

Apparently, there are no mentions about directionality constraints in power transmission, but it needs to be investigated more.

\section{HEATING}

The majority of the implantable devices are packed in a metallic case, as titanium, to ensure the hermeticity and the biocompatibility. Whereas the negative aspect of using metallic enclosures for medical implants involves the formation of superficial currents, which imply a heating of the device's casing with the consequent tissues heating. These superficial electrical currents are defined as Eddy current, they are induced in a conductor by an incident and variable magnetic field, due to Faraday's law of induction. They flow in closed loops on conductor's surface, in planes perpendicular to the magnetic field [18].

This is the most relevant issue in the wireless power transmission. Through the inductive technique, it is possible to optimise the system, in order to reduce this effect. So studying the geometry, number and material of turns and employing a ferrite core in Tx coil. All these parameters can reduce the heating, but they cannot delete it.

From the point of view of magnetostrictive materials, there are no mentions in the literature about this issue. It could be interesting to analyse more this aspect and compare it to the previous system, in order to verify the applicability for the medical regulatory.

\section{CONCLUSION}

A novel technology, based on magnetoelectric system, is presented. An artificial magnetoelectric materials can be configured to efficiently convert AC magnetic electric fields to electrical power, at electromechanical resonance frequency. This could be an alternative system to wirelessly transmit power for battery-less AIMD; because it does not depend on directionality/alignment of the external coil with the internal receiver, as the two-coils system requires.

\section{ACKNOWLEDGEMENT}

This work is funded by the European Union's H2020: MSCA: ITN program for the "Wireless In-body Environment Communication - WiBEC" project under the grant agreement no. 675353 .

\section{REFERENCES}

[1] Zurbuchen A. et al, Towards Batteryless Cardiac Implantable Electronic Devices - The Swiss Way

[2] Lee J. et al, A review on Wireless Powering Schemes for Implantable Microsystems in Neural Engineering Application

[3] Radziemski L., Makin I., In vivo demonstration of ultrasound power delivery to charge implanted medical devices via acute and survival porcine studies

[4] Abiri P. et al, Inductively powered wireless pacing via a miniature pacemaker and remote stimulation control system

[5] ICNIRP Guidelines

[6] Lafont T., Magnetostrictive-piezoelectric composite structures for energy harvesting

[7] Lee J. et al, A review on Wireless Powering Schemes for Implantable Microsystems in Neural Engineering Application

[8] Bozorth R., Ferromagnetism

[9] Yang G., Contribution to modelling of magnetoelectric composites for energy harvesting

[10] Palaksha D. and Rizzo G. et al, Load modulation for leadless pacemaker synchronization in a dual chamber pacemaker system 
[11] Yin M. et al, A 100-Channel hermetically sealed implantable device for chronic wireless neurosensing applications

[12] Yang G., Contribution to modelling of magnetoelectric composites for energy harvesting

[13] Wang Y. et al, Electrical resistance load effect on magnetoelectric coupling of magnetostrictivelpiezoelectric laminated composite

[14] Dong S., Longitudinal and Transverse Magnoelectric Voltage Coefficients of Magnetostrictive/Piezoelectric Laminate Composite: Theory
[15] Malleron K et al., Experimental study of magnetoelectric transducers for power supply of small biomedical devices

[16] Filippov D.A. et al, Resonance magnetoelectric effects in magnetostrictive-piezoelectric three-layer structures

[17] O'Handley R.C. et al, Improved Wireless, Transcutaneous Power Transmission for In Vivo Applications

[18] Umenei A.E.et al, Novel Method for Selective Non-linear Fluxguide Switching for Contacless Inductive Power Transfer 